\title{
Impact of core-shell dipolar interaction on magnetic phases of spherical core-shell nanoparticles
}

\author{
F. C. Medeiros Filho, L. L. Oliveira, S. S. Pedrosa, G. O. G. Rebouças, and A. S. Carriço* \\ Departamento de Física, Universidade Federal do Rio Grande do Norte, 59072-970 Natal, Rio Grande do Norte, Brazil
}

Ana L. Dantas

Departamento de Física, Universidade do Estado do Rio Grande do Norte, 59610-210 Mossoró, Rio Grande do Norte, Brazil

(Received 27 May 2015; revised manuscript received 29 July 2015; published 26 August 2015)

\begin{abstract}
We show that confinement in small volumes affects the interplay of exchange and dipolar interactions and the magnetic phases of hard and soft spherical core-shell nanoparticles. Large variations in the magnetization of thin shells may occur due to the core dipolar field gradient within the shell. The reversal field is tunable by the trends imposed by the dipolar and core-shell interface exchange energies. We show, for instance, that the reversal field of a $\mathrm{CoFe}_{2} \mathrm{O}_{4}(30 \mathrm{~nm}) @ \mathrm{MnFe}_{2} \mathrm{O}_{4}(6 \mathrm{~nm})$ particle ranges from $15.5 \mathrm{kOe}$ for antiferromagnetic coupling down to $2.5 \mathrm{kOe}$ for ferromagnetic coupling.
\end{abstract}

DOI: 10.1103/PhysRevB.92.064422

PACS number(s): 75.75.Fk, 75.60.-d

\section{INTRODUCTION}

Bimagnetic core-shell nanoparticle systems are currently the focus of a great deal of research effort [1]. From the applications viewpoint, the motivation is the chance of combining different functionalities of two magnetic materials into the effective magnetic properties of a single nanoparticle. From the standpoint of fundamental magnetism, there is interest in new magnetic phases that may emerge from the intrinsic properties of the core and shell materials and the core-shell interactions.

Early studies focused on the magnetic properties of nanoparticles made of ferromagnetic (FM) and antiferromagnetic (AFM) materials [1], motivated by the enormous impact of the exchange-bias phenomenology [2,3] in the design of spin-valve systems [4] for giant magnetoresistance sensors [5] and tunneling devices [6,7].

The large variety of interesting phenomena reported so far for core-shell nanoparticles systems made of FM or ferrimagnetic (FiM) and AFM materials has motivated Monte Carlo model studies of the exchange-bias phenomenology of small nanoparticles, with a few thousand [8-14] or a few hundred thousand [15] magnetic moments and short-range (anisotropy and exchange) interactions.

Current applications, such as core-shell nanoparticles for high-energy-product permanent magnets [16-19] and for biomedical applications [20-23], demand theoretical modeling for nanoparticle systems with dimensions ranging from 10 to $100 \mathrm{~nm}$. In this size range, FM or FiM nanoparticles may have over $10^{7}$ magnetic moments, which are subjected to both short-range (exchange and anisotropy) and long-range (dipolar) interactions.

With current standard computational facilities, the study of the magnetic phases and switching processes of nanoparticles with dimensions in the sub-100-nm size range, based on the investigation of the behavior of their individual magnetic moments using, for instance, the Monte Carlo technique, becomes prohibitively time-consuming.

These computational difficulties affect the theoretical analysis of the intraparticle dipolar interaction and the interparticle

\footnotetext{
*ascarrico@gmail.com
}

dipolar interaction. Approximations have been proposed to set up theoretical models for the competition between the exchange and dipolar energies within a single particle and the interparticle interactions in systems with a large number of small nanoparticles [24-26].

A fast Monte Carlo method has been proposed [24] in order to overcome these size restrictions and incorporate the longrange dipolar interaction for the investigation of the magnetic phases of a single material cubic ferromagnetic particle.

This method is an approximation based on the Monte Carlo method and combines the standard Monte Carlo method with scaling techniques to allow investigation of the magnetic properties of ferromagnetic nanoparticles with a few million magnetic moments. The predictions of the micromagnetic theory [27] regarding the magnetic phases of ferromagnetic cubic particles have been reproduced and used as a check to validate the fast Monte Carlo method results.

Theoretical models representing interparticle interactions in systems with a large number of small FM/AFM core-shell nanoparticles have been proposed to investigate the basic phenomenology of exchange bias $[25,26]$. Each nanoparticle is represented by a pair of exchange-coupled, anisotropic spins [25], one for the core and another for the shell, or a single spin for the core and a pair of spins for the shell [26].

These models assume coherent rotation of the core and shell magnetization, which is a reasonable first approximation for small-diameter core-shell nanoparticles. The interparticle dipolar interaction between a large number of core-shell nanoparticles is investigated using a smaller set of nanoparticle placed in two-dimensional periodic arrays, together with cyclic boundary conditions. The dipolar sums are calculated using the Ewald summation method [28].

In order to handle a large number of magnetic moments subjected to long-range dipolar interactions, one possibility is to operate on a larger scale by regarding the particle as being composed of small units, which are nevertheless large enough to allow a continuous approximation of their magnetization. This is the basis of the micromagnetism approach [29], which has been largely used to determine the magnetic phases, such as domain walls and vortices, and reversal mechanisms of magnetic particles, with dimensions in the sub-100-nm up to micrometer range [30-35]. 
A recent micromagnetic study of hard/soft core-shell nanoparticles, with modest magnetization shells, with saturation magnetization Ms from 250 to $500 \mathrm{emu} / \mathrm{cm}^{3}$, revealed a Stoner-Wohlfarth behavior for the core-shell nanoparticle hysteresis loops [36]. In this case, due to the small value of the shell magnetization, dipolar effects are weak, and the angular dependence of the switching field follows the Stoner-Wohlfarth reversal mode [37].

Applications based on hard/soft core-shell (HSCS) nanoparticles, such as systems for permanent magnets and magnetic hyperthermia, require strong shell-to-core pinning and coherent reversal. One may think that strong ferromagnetic core-shell interface exchange energy and shell thicknesses smaller than the shell exchange length might grant shell-tocore pinning and coherent reversal. This intuitive idea has been confirmed by a number of experimental reports [1] and has motivated detailed studies of the magnetic phases and reversal processes of bimagnetic core-shell nanoparticles and nanocomposites [1,38], focusing either on structural magnetic parameters, such as the nature of the core-shell exchange interaction, or on the effective magnetic parameters of the core-shell nanoparticles, such as the coercivity and the magnetization.

Most often, small-diameter hard/soft core-shell nanoparticles exhibit smooth hysteresis curves, as expected for strong ferromagnetic core-shell exchange energy. However, a recent report [39] on core-shell nanoparticles based on iron and manganese oxides revealed that the interface coupling is antiferromagnetic, with positive exchange-bias shift, as reported much earlier for antiferromagnetically coupled FM/AFM bilayers [40].

The magnetization of small-diameter hard/soft core-shell nanoparticles increases, and the coercive field is monotonically decreased as the soft-phase content is increased [41-43]. This corresponds to smooth hysteresis loops, in which the magnetic behaviors of the core and the shell are not distinguishable, indicating that core-shell materials are exchange coupled. Two-phase hysteresis loops suggesting independent reversal of the soft material have also been reported [44-46] and attributed to the large size of the soft material, weak interface exchange coupling, or interparticle dipolar interaction.

Here we discuss features arising from the core-shell dipolar interaction that may affect the magnetic phases and reversal mechanism of HSCS nanoparticles with large magnetization shells. Our results suggest that the core dipolar field may have a large impact on the shell magnetic phases, triggering early shell reversal and reducing the field for core-shell reversal. On the other hand for large magnetization thick shells, the shell dipolar field may be along the core magnetization direction, leading to further stabilization of the core.

In addition, we show that the core-shell dipolar interaction favors an antiferromagnetic arrangement of the shell and core magnetizations, and ferromagnetic (antiferromagnetic) interface exchange energy leads to reduction (enhancement) of the core-shell reversal field. The impact of the core-shell dipolar interaction on the magnetic phases is greatly enhanced by the geometrical constraints and the magnetic properties of the HSCS materials. The large anisotropy qualifies the core as a stable dipolar field source. Furthermore, there is a large variation of the core dipolar field in the shell on a

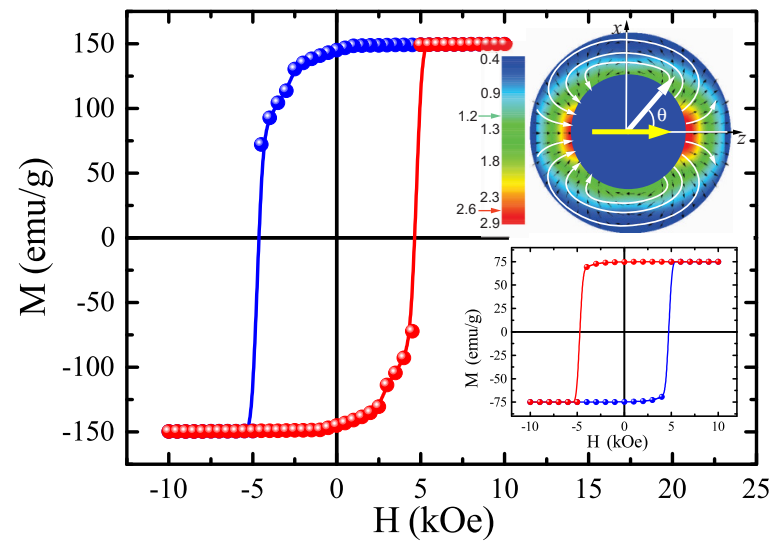

FIG. 1. (Color online) Hysteresis curve of a $\mathrm{CoFe}_{2} \mathrm{O}_{4}(22 \mathrm{~nm})$ $@ \mathrm{CoFe}_{2}(2 \mathrm{~nm})$ nanoparticle and of the $\mathrm{CoFe}_{2} \mathrm{O}_{4}(22 \mathrm{~nm})$ core (in the bottom inset). The top inset shows the $\mathrm{CoFe}_{2} \mathrm{O}_{4}$ core dipolar field on the $\mathrm{CoFe}_{2}$ shell, and the color bar indicates the dipolar field strength.

length scale of the order of the core radius. The core dipolar field in the shell is parallel to the core magnetization near the polar regions $(|\delta \theta| \approx \pi / 4$, around the $\theta=0$ and $\theta=\pi$ poles) and opposite the core magnetization at the $\theta=\pi / 2$ belt (see Fig. 1). This built-in dipolar field gradient favors a nonuniform magnetization profile at the shell. One may expect larger effects for large core diameters and large magnetization shell materials.

Neglecting deviations of the core magnetization from the uniaxial axis, the core dipolar field on the shell may be estimated from the field of a point dipole with a magnetic moment of $\mu=4 \pi r^{3} M_{S} / 3$. The dipolar field strength at the poles is $8 \pi M_{S} / 3$, and at the $\theta=\pi / 2$ belt it is $4 \pi M_{S} / 3$, in the opposite direction (see bottom inset in Fig. 1). For a $\mathrm{CoFe}_{2} \mathrm{O}_{4}$ core, this amounts to a change in the $z$-axis field of the order of $5 \mathrm{kOe}$ on a length scale of the order the radius $r$.

We have chosen nanoparticles composed of a hard $\mathrm{CoFe}_{2} \mathrm{O}_{4}$ core and soft $\mathrm{MnFe}_{2} \mathrm{O}_{4}$ and $\mathrm{CoFe}_{2}$ shells. $\mathrm{CoFe}_{2}$ magnetization $(228 \mathrm{emu} / \mathrm{g})$ is larger than that of $\mathrm{MnFe}_{2} \mathrm{O}_{4}(80 \mathrm{emu} / \mathrm{g})$.

The motivation for choosing two materials with a large difference in the magnetization is that one may investigate the impact of large magnetization shell materials on the core-shell dipolar interaction and on the effective magnetic parameters of HSCS nanoparticles. $\mathrm{MnFe}_{2} \mathrm{O}_{4}$ and $\mathrm{CoFe}_{2}$ have densities [47] of 5.0 and $8.0 \mathrm{~g} / \mathrm{cm}^{3}$. Therefore for a given value of the shell volume, a $\mathrm{CoFe}_{2}$ shell has a magnetic moment more than four times larger than the corresponding value for $\mathrm{MnFe}_{2} \mathrm{O}_{4}$ shell. Furthermore, as we shall discuss below, variations in the direction of the magnetization in the $\mathrm{CoFe}_{2}$ shell leads to interesting effects associated with the dipolar field produced by the shell in the core.

\section{THEORETICAL MODEL}

The magnetic structure is described using small cubic cells, with the edge smaller than the domain-wall width and exchange lengths of the core and shell materials. The $z$ axis is chosen to be along the uniaxial anisotropy of the $\mathrm{CoFe}_{2} \mathrm{O}_{4}$ 
core. The energy density is given by

$$
\begin{aligned}
E= & -H \hat{z} \sum_{j} M_{S}^{j} \hat{m}_{j}-\sum_{j} K^{j}\left(m_{j}^{z}\right)^{2} \\
& +\frac{1}{2} \sum_{j} \sum_{k} M_{S}^{j} M_{S}^{k}\left(\frac{\hat{m}_{j} \hat{m}_{k}}{n_{j k}^{3}}-\frac{3\left(\hat{m}_{j} \hat{n}_{j k}\right)\left(\hat{m}_{k} \hat{n}_{j k}\right)}{n_{j k}^{5}}\right) \\
& +\frac{1}{d^{2}} \sum_{j} \sum_{k} A_{j k}\left(1-\hat{m}_{j} \hat{m}_{k}\right) .
\end{aligned}
$$

The first two terms are the Zeeman and anisotropy energies, and $M_{S}^{j}$ and $\hat{m}_{j}$ are the $j$-cell saturation magnetization value and direction. In the dipolar energy, $n_{j k}$ is the distance between cells $j$ and $k$ in units of cell edge. The exchange energy couples nearest-neighbor cells. $A_{j k}$ is either the core or the shell exchange stiffness for cells $j$ and $k$ within the same material; otherwise, it represents the effective interface exchange energy. For $\mathrm{CoFe}_{2} \mathrm{O}_{4}$ we use $M_{S}=75 \mathrm{emu} / \mathrm{g}$ [48], $A=3.2 \times 10^{-12} \mathrm{~J} / \mathrm{m}$ [48], and $K=2.7 \times 10^{5} \mathrm{~J} / \mathrm{m}^{3}$ [49]. For $\mathrm{MnFe}_{2} \mathrm{O}_{4}[50]$ we use $M_{S}=80 \mathrm{emu} / \mathrm{g}, A=3.2 \times 10^{-12} \mathrm{~J} / \mathrm{m}$, and $K=2.5 \times 10^{3} \mathrm{~J} / \mathrm{m}^{3}$. For $\mathrm{CoFe}_{2}$ we use $M_{S}=228 \mathrm{emu} / \mathrm{g}$ [51], $A=1.9 \times 10^{-12} \mathrm{~J} / \mathrm{m}$ [17], and $K=1.7 \times 10^{4} \mathrm{~J} / \mathrm{m}^{3}$ [52].

At the interface, the local environment of the magnetic ions is affected by composition gradients and interface roughness, and the core-shell exchange interaction depends on sample preparation [53-55]. We make the simplifying assumption of a uniform interface and use an effective exchange parameter $A^{*}$, which measures the interface exchange energy in units of the $\mathrm{CoFe}_{2} \mathrm{O}_{4}$ core exchange stiffness.

The equilibrium configuration is found using numeric procedures which allow finding the magnetic patterns in a self-consistent manner [56]. At each cell, the magnetization direction $\hat{m}_{j}$ is adjusted to be parallel to the local magnetic field $\left[H_{\mathrm{eff}}^{j}=-\left(1 / M_{S}\right)\left(\partial E / \partial \hat{m}_{j}\right)\right]$, so that within a reasonable numerical precision, the torque is zero, $\vec{m}_{j} \times \vec{H}_{\text {eff }}^{j}=0$. Convergence is checked to guarantee a maximum torque of $10^{-27} \mathrm{~J}$.

\section{RESULTS AND DISCUSSION}

In Fig. 1 we show the hysteresis of a $\mathrm{CoFe}_{2} \mathrm{O}_{4}(22$ $\mathrm{nm}) @ \mathrm{CoFe}_{2}(2 \mathrm{~nm})$ nanoparticle for an interface exchange energy of the order of the $\mathrm{CoFe}_{2} \mathrm{O}_{4}$ exchange stiffness $\left(A^{*}=\right.$ $0.8)$. At the reversal field the magnetization has dropped to $50 \%$ of the saturation value, corresponding to the $\mathrm{CoFe}_{2}$ shell magnetization dropping from saturation down to $35 \%$ of the saturation value. The $\mathrm{CoFe}_{2} \mathrm{O}_{4}$ core has a square hysteresis loop, and the continuous variation of the core-shell magnetization is due to the early shell reversal. This sounds surprising for a thin 2-nm shell with strong ferromagnetic core-shell exchange coupling.

The $\mathrm{CoFe}_{2} \mathrm{O}_{4}$ core has a square hysteresis, providing the key mechanism for early shell reversal and reduction of the core-shell coercivity. The core-shell reversal is driven by the local field in most of the shell volume. Except for the polar regions $(|\delta \theta| \approx \pi / 4$, around the $\theta=0$ and $\theta=\pi$ poles) the core dipolar field is opposite the core magnetization. Furthermore, at the upper branch of the hysteresis curve, when

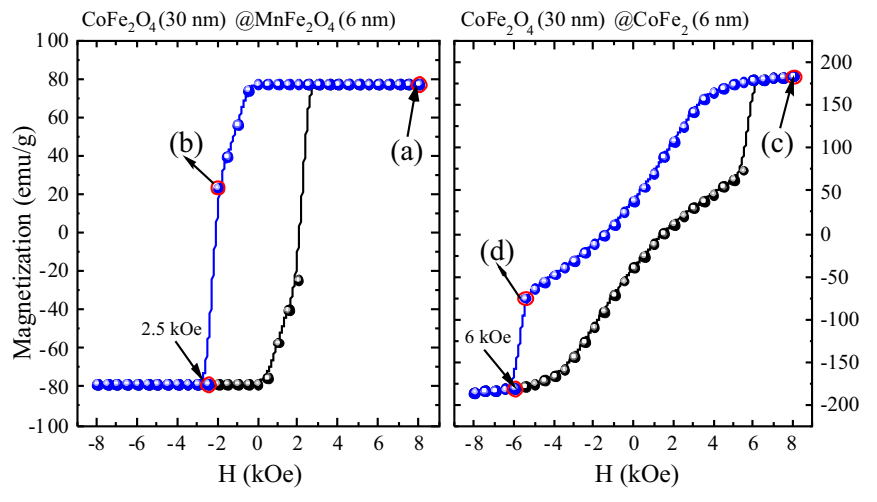

FIG. 2. (Color online) Hysteresis curves of 30-nm-diameter $\mathrm{CoFe}_{2} \mathrm{O}_{4}$ core nanoparticles with 6-nm-thick (left) $\mathrm{MnFe}_{2} \mathrm{O}_{4}$ and (right) $\mathrm{CoFe}_{2}$ shells for strong interface exchange energy.

$H<0$, the external field is in the direction of the core dipolar field in the $\theta=\pi / 2$ belt. At the reversal field ( $H \approx-5 \mathrm{kOe}$ ), the $z$ component of the core dipolar field in the shell varies from $H_{z}^{\text {core }}=2.68 \mathrm{kOe}$ at the poles to $H_{z}^{\text {core }}=-1.23 \mathrm{kOe}$ at the $\theta=\pi / 2$ belt. Thus the local field at the $\theta=\pi / 2$ belt is opposite the core magnetization, with a strength of $\approx 6 \mathrm{kOe}$. The strong ferromagnetic coupling favors the alignment of the core and shell magnetizations, and the core-shell reversal occurs at an external field strength $(5 \mathrm{kOe})$ much smaller than the core uniaxial anisotropy field $(13.5 \mathrm{kOe})$.

The hysteresis curves of $\mathrm{CoFe}_{2} \mathrm{O}_{4}(30 \mathrm{~nm}) @ \mathrm{MnFe}_{2} \mathrm{O}_{4}(6$ $\mathrm{nm})$ and $\mathrm{CoFe}_{2} \mathrm{O}_{4}(30 \mathrm{~nm}) @ \mathrm{CoFe}_{2}(6 \mathrm{~nm})$ nanoparticles (see Figs. 2 and 4) reveal interesting features. The shell volume fraction is $64 \%$, and there is a large impact of the $\mathrm{CoFe}_{2}$ shell magnetic patterns on the $\mathrm{CoFe}_{2} \mathrm{O}_{4}(30 \mathrm{~nm}) @ \mathrm{CoFe}_{2}(6 \mathrm{~nm})$ magnetic phases.

With strong ferromagnetic coupling $\left(A^{*}=0.8\right)$, there is a considerable reduction of the reversal field, as shown in Fig. 2. The $\mathrm{MnFe}_{2} \mathrm{O}_{4}$ shell leads to a much smaller reversal field ( $2.5 \mathrm{kOe})$ than the $\mathrm{CoFe}_{2}$ shell $(6 \mathrm{kOe})$. Intuitively, one would expect the opposite to occur since $\mathrm{CoFe}_{2}$ has a much larger magnetization. However, as shown in the dipolar field maps in Fig. 3, there are relevant differences in the dipolar field produced by the $\mathrm{CoFe}_{2}$ and $\mathrm{MnFe}_{2} \mathrm{O}_{4}$ shells in the $\mathrm{CoFe}_{2} \mathrm{O}_{4}$ core.

The sources of shell dipolar field are the volume density of magnetic charges and the unbalanced magnetic surface charges at the shell surfaces and are much larger for the $\mathrm{CoFe}_{2}$ shell. The $\mathrm{MnFe}_{2} \mathrm{O}_{4}$ shell produces a small field parallel to the core magnetization, as seen in the dipolar field panels in Figs. 3(a) and 3(b). On the other hand, changes in the $\mathrm{CoFe}_{2}$ shell magnetic pattern lead to considerable changes in the dipolar field produced in the core. At $H=8 \mathrm{kOe}$, with the shell magnetization nearly aligned with the core [Fig. 3(c)], the shell dipolar field in the core is small and opposite the core magnetization. At the reversal field, the $\mathrm{CoFe}_{2}$ shell produces a dipolar field in the direction of the core magnetization of the order of $3 \mathrm{kOe}$ in most of the core volume, reaching around $5 \mathrm{kOe}$ near the poles [Fig. 3(d)]. The core-shell dipolar interaction compensates the increase in the Zeeman energy, leading to a reversal field of $6 \mathrm{kOe}$. 

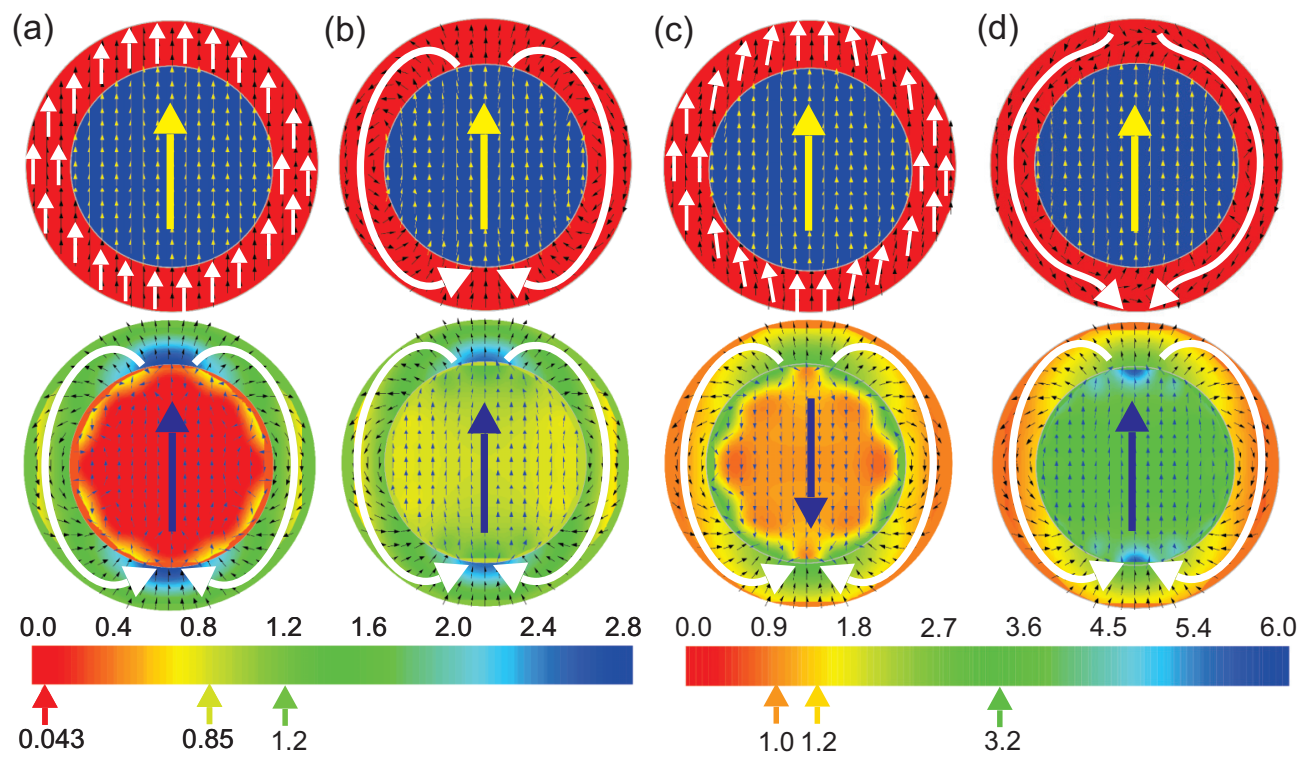

FIG. 3. (Color online) Magnetic pattern (top panels) and dipolar field maps of 30-nm-diameter $\mathrm{CoFe}_{2} \mathrm{O}_{4}$ core nanoparticles with 6-nm-thick (a) and (b) $\mathrm{MnFe}_{2} \mathrm{O}_{4}$ and (c) and (d) $\mathrm{CoFe}_{2}$ shells at selected points of the hysteresis curves shown in Fig. 2. The color bar indicates the dipolar field strength (in kOe) and applies to both the shell dipolar field in the core and the core dipolar field in the shell.

For $A^{*}=0$ (see Fig. 4), the core-shell dipolar interaction leads to reversal field values larger than the anisotropy field of the $\mathrm{CoFe}_{2} \mathrm{O}_{4}$ core. The average core dipolar field on the $\theta \approx$ $\pi / 2$ belt at the shell has an inverse hysteresis [see Fig. 5(a)] and has a larger impact in the $\mathrm{CoFe}_{2}$ shell. The $\mathrm{MnFe}_{2} \mathrm{O}_{4}$ shell starts switching at $1.5 \mathrm{kOe}$ with a remanence of $-23.17 \mathrm{emu} / \mathrm{g}$, while the $\mathrm{CoFe}_{2}$ shell starts switching earlier, at $H=15 \mathrm{kOe}$, with a remanence of $-30 \mathrm{emu} / \mathrm{g}$.

The average of the $\mathrm{CoFe}_{2}$ shell dipolar field in the $\mathrm{CoFe}_{2} \mathrm{O}_{4}$ core also displays an inverted hysteresis [see Fig. 5(b)]. Changes in the shell magnetization pattern produce large variation of the shell dipolar field. As shown in panels (i) (for
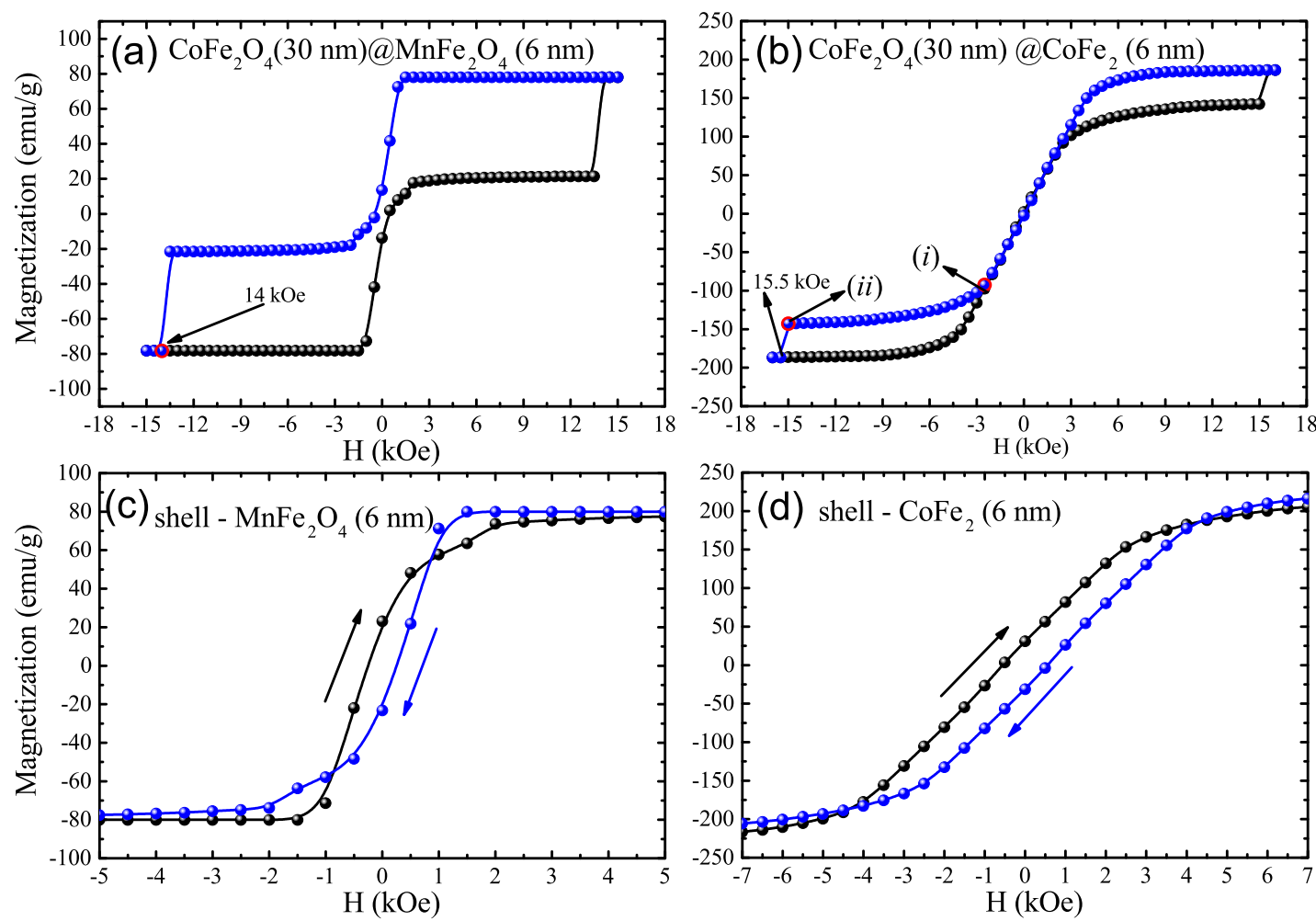

FIG. 4. (Color online) Hysteresis curves of 30-nm-diameter $\mathrm{CoFe}_{2} \mathrm{O}_{4}$ core nanoparticles with 6-nm-thick (a) $\mathrm{MnFe}_{2} \mathrm{O}_{4}$ and (b) $\mathrm{CoFe} e_{2}$ shells and the inverted hysteresis of the (c) $\mathrm{MnFe}_{2}$ and (d) $\mathrm{CoFe}_{2}$ shells in the absence of interface exchange energy $\left(A^{*}=0\right)$. 

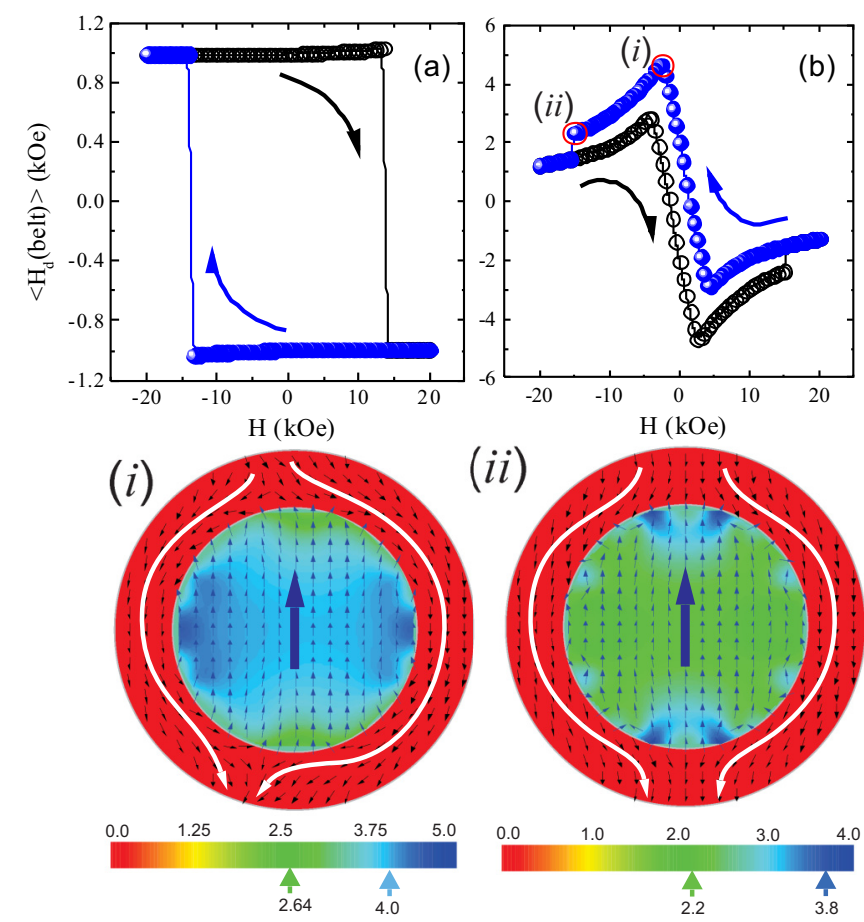

FIG. 5. (Color online) (a) Average of the 30-nm-diameter $\mathrm{CoFe}_{2} \mathrm{O}_{4}$ core dipolar at the $\theta \cong \pi / 2$ belt at the 6-nm-thick $\mathrm{CoFe}_{2}$ shell. (b) Average of the dipolar field of the 6-nm-thick $\mathrm{CoFe}_{2}$ shell on the 30-nm-diameter $\mathrm{CoFe}_{2} \mathrm{O}_{4}$ core. The close-ups (i) and (ii) show the shell magnetic pattern and the dipolar field produced in the core for selected points of the hysteresis shown in Fig. 4(b). The color bar gives the intensity of the dipolar field (in kOe).

$H=-3 \mathrm{kOe}$ ) and (ii) (for $H=-15.5 \mathrm{kOe}$ ) in Fig. 5(b), the alignment of the shell magnetization with the external field produces a drop from 4.5 down to $2 \mathrm{kOe}$ in the average shell dipolar field.

Ferromagnetic (antiferromagnetic) interface exchange energy leads to a reduction (enhancement) of the core-shell reversal field. As shown in Fig. 6 for a 30-nm-diameter $\mathrm{CoFe}_{2} \mathrm{O}_{4}$ core and 6-nm-thick $\mathrm{MnFe}_{2} \mathrm{O}_{4}$ shell, the reversal field ranges from $15.5 \mathrm{kOe}$ for antiferromagnetic coupling down to $2.5 \mathrm{kOe}$ for ferromagnetic coupling.

We note two interesting features. First, for $A^{*}>0.3$, the reversal field for the $\mathrm{CoFe}_{2} \mathrm{O}_{4}(30 \mathrm{~nm}) @ \mathrm{MnFe}_{2} \mathrm{O}_{4}(6 \mathrm{~nm})$ core-shell nanoparticle is $3 \mathrm{kOe}$ smaller than that for a $\mathrm{CoFe}_{2} \mathrm{O}_{4}(30 \mathrm{~nm}) @ \mathrm{CoFe}_{2}(6 \mathrm{~nm})$ core-shell nanoparticle. Second, in the inset of Fig. 6, the nearly linear decay of the reversal field for $\mathrm{MnFe}_{2} \mathrm{O}_{4}(\delta \mathrm{nm})$ shells corresponds to common intuition since the effective magnetization increases with shell thickness. $\mathrm{CoFe}_{2}(\delta \mathrm{nm})$ shells show a striking difference. The reversal field of the $\mathrm{CoFe}_{2} \mathrm{O}_{4}(30 \mathrm{~nm}) @ \mathrm{CoFe}_{2}(\delta \mathrm{nm})$ core-shell nanoparticles is nearly constant for shell thicknesses ranging from 2 to $8 \mathrm{~nm}$. Both features are due to the core stabilization due to the dipolar field produced by the $\mathrm{CoFe}_{2}(6 \mathrm{~nm})$ shell parallel to the magnetization of the $\mathrm{CoFe}_{2} \mathrm{O}_{4}(30 \mathrm{~nm})$ core.

For small-diameter core-shell nanoparticles and strong core-to-shell pinning, one might expect coherent reversal, and using average magnetic parameters [57], the coercivity should be given by $H_{c}=\frac{2\langle K\rangle}{\langle M\rangle} \cdot \mathrm{CoFe}_{2} \mathrm{O}_{4}(9 \mathrm{~nm}) @ \mathrm{MnFe}_{2} \mathrm{O}_{4}(3 \mathrm{~nm})$

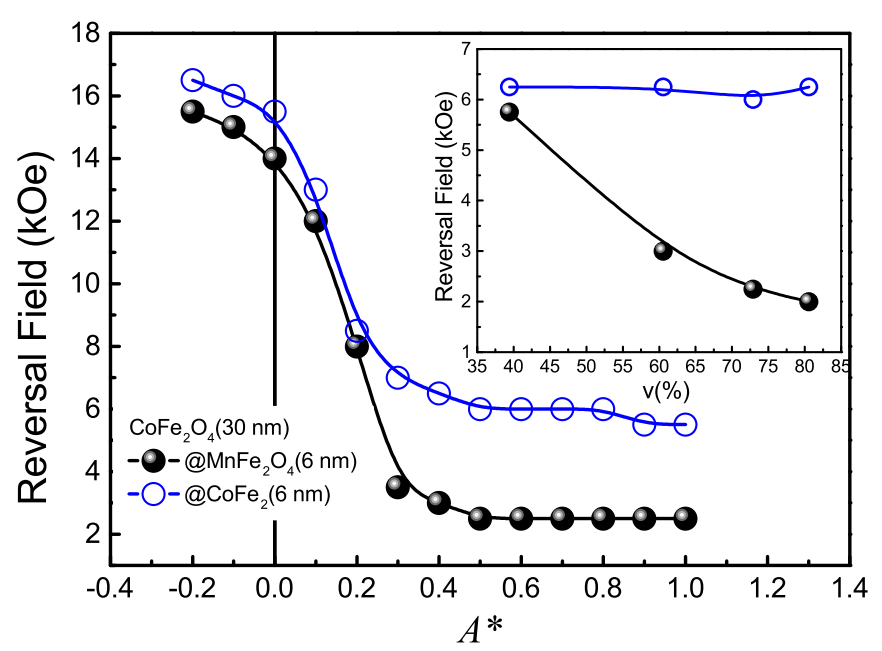

FIG. 6. (Color online) Reversal field of $\mathrm{CoFe}_{2} \mathrm{O}_{4}(30 \mathrm{~nm})$ @ $\mathrm{MnFe}_{2} \mathrm{O}_{4}(6 \mathrm{~nm})$ (curve with solid circles) and $\mathrm{CoFe}_{2} \mathrm{O}_{4}(30 \mathrm{~nm})$ $@ \mathrm{CoFe}_{2}(6 \mathrm{~nm})$ (curve with open circles) nanoparticles. The inset shows the reversal field of $\mathrm{CoFe}_{2} \mathrm{O}_{4}(30 \mathrm{~nm}) @ \mathrm{MnFe}_{2} \mathrm{O}_{4}(\delta \mathrm{nm})$ and $\mathrm{CoFe}_{2} \mathrm{O}_{4}(30 \mathrm{~nm}) @ \mathrm{CoFe}_{2}(\delta \mathrm{nm})$ for $A^{*}=0.8$ and $\delta=2,4,6$, and $8 \mathrm{~nm}$, corresponding to a shell volume fraction ranging from $30 \%$ to $73 \%$

and $\mathrm{CoFe}_{2} \mathrm{O}_{4}(6 \mathrm{~nm}) @ \mathrm{MnFe}_{2} \mathrm{O}_{4}(2 \mathrm{~nm})$ have the same core and shell volume fractions of $f_{C}=0.22$ and $f_{S}=0.78$. As shown in Table I, using average magnetic parameters, the coercivity should be $3.0 \mathrm{kOe}$ for both nanoparticles. However, the measured coercivity of $\mathrm{CoFe}_{2} \mathrm{O}_{4}(6 \mathrm{~nm}) @ \mathrm{MnFe}_{2} \mathrm{O}_{4}(2 \mathrm{~nm})$ is much larger $(9.7 \mathrm{kOe})$ [41]. This nanoparticle has a smaller diameter and a thinner shell, with a larger chance of interface defects and reduced interface exchange energy. We have reproduced the experimental data using a small interface energy $\left(A^{*}=0.025\right)$.

In summary, we have investigated the impact of the coreshell dipolar interaction on the magnetic phases and reversal mechanism of $\mathrm{CoFe}_{2} \mathrm{O}_{4} @ \mathrm{MnFe}_{2} \mathrm{O}_{4}$ and $\mathrm{CoFe}_{2} \mathrm{O}_{4} @ \mathrm{CoFe}_{2}$ core-shell nanoparticles. We have focused on the impact of the core-shell exchange energy and the core-shell dipolar interaction on the magnetic phases and on the reversal field. We have found that ferromagnetic (antiferromagnetic) coreshell exchange coupling leads to a reduction (enhancement) of the core-shell reversal field. As might be expected, for ferromagnetic exchange coupling, the reduction becomes larger as the core-shell exchange energy is increased.

We note that considering the core and shell materials as separate magnetic units interacting via exchange and dipolar energies allows us to investigate the impact of the geometrical parameters and the strength of the core-shell interaction in the

TABLE I. Coercive field of selected small-diameter core-shell nanoparticles.

\begin{tabular}{lccc}
\hline \hline \multirow{2}{*}{ System } & \multicolumn{3}{c}{$H_{c}(\mathrm{kOe})$} \\
\cline { 2 - 4 } & Exp. & Theory & $\frac{2\langle K\rangle}{\langle M\rangle}$ \\
\hline $\mathrm{CoFe}_{2} \mathrm{O}_{4}(9 \mathrm{~nm}) @ \mathrm{MnFe}_{2} \mathrm{O}_{4}(3 \mathrm{~nm})$ & $2.53[21]$ & 2.6 & 3.0 \\
$\mathrm{CoFe}_{2} \mathrm{O}_{4}(6 \mathrm{~nm}) @ \mathrm{MnFe}_{2} \mathrm{O}_{4}(2 \mathrm{~nm})$ & $9.7[41]$ & 9.0 & 3.0 \\
\hline \hline
\end{tabular}


reversal process. As shown in the example given in Table I, the strength of the interface coupling may lead to quite different reversal fields for core-shell nanoparticles made of the same materials with the same core and volume fractions but different core and shell sizes.

An interesting feature emerges from the comparison of the dependence of the reversal field on the strength of the interface exchange energy for core-shell nanoparticles composed of a 30-nm $\mathrm{CoFe}_{2} \mathrm{O}_{4}$ core and 6-nm-thick $\mathrm{MnFe}_{2} \mathrm{O}_{4}$ and $\mathrm{CoFe}_{2}$ shells. As shown in Fig. 6, for strong ferromagnetic coupling, the reversal field of $\mathrm{CoFe}_{2} \mathrm{O}_{4} @ \mathrm{CoFe}_{2}$ nanoparticles is $3 \mathrm{kOe}$ larger. We attribute this difference to the stabilization of the $\mathrm{CoFe}_{2} \mathrm{O}_{4}$ core by the dipolar field produced by the large magnetization $\mathrm{CoFe}_{2}$ shell.

The core-shell dipolar interaction may be viewed in a simple manner. The core produces a dipolar field in the shell, which, as depicted in the top inset of Fig. 1, is parallel to the core magnetization in the shell region near the poles of the core and is oriented opposite the core magnetization at the $\theta=\pi / 2$ belt.

This dipolar field pattern competes with the ferromagnetic core-shell exchange coupling, favoring the nucleation of a nonuniform magnetization pattern in the shell, like an onion state [see the top panels in Figs. 3(b) and 3(d)]. At the demagnetization quadrant a large fraction of the shell volume is magnetized in the direction of the external field, favoring the reversal of the core-shell nanoparticle at an external field value smaller than that predicted by the average core-shell model [57].

Furthermore, the shell also produces a dipolar field in the core. The sources of the shell dipolar field are the volume density of magnetic charges and the unbalanced magnetic surface charges at the shell surfaces. As shown in the bottom panels of Fig. 3, $\mathrm{CoFe}_{2}$ shells produces larger dipolar fields in the $\mathrm{CoFe}_{2} \mathrm{O}_{4}$ core.

We have found that the built-in $\mathrm{CoFe}_{2} \mathrm{O}_{4}$ dipolar field affects the magnetic phases by favoring early shell reversal. This leads to a reduction of the core-shell reversal field of $\mathrm{CoFe}_{2} \mathrm{O}_{4} @ \mathrm{MnFe}_{2} \mathrm{O}_{4}$ nanoparticles. For $\mathrm{CoFe}_{2} \mathrm{O}_{4} @ \mathrm{CoFe}_{2}$ nanoparticles, the dipolar field produced by the $\mathrm{CoFe}_{2}$ shell produces a stabilization of the $\mathrm{CoFe}_{2} \mathrm{O}_{4}$ core magnetization, attenuating the reduction of the core-shell reversal field.

$\mathrm{CoFe}_{2} \mathrm{O}_{4} @ \mathrm{MnFe}_{2} \mathrm{O}_{4}$ core-shell nanoparticles almost correspond with the intuitive behavior predicted by the average core-shell model, with smooth hysteresis loops and a nearly linear decay of the reversal field with the $\mathrm{MnFe}_{2} \mathrm{O}_{4}$ volume fraction, as expected for strong core-shell exchange coupling.

The $\mathrm{CoFe}_{2} \mathrm{O}_{4}(30 \mathrm{~nm}) @ \mathrm{MnFe}_{2} \mathrm{O}_{4}(6 \mathrm{~nm})$ nanoparticle exhibits a nearly square hysteresis loop, with a small signature of early shell reversal. In this case the coercive field $(2.5 \mathrm{kOe})$ is smaller than the coercive field predicted by the average core-shell model (4.8 kOe).

$\mathrm{CoFe}_{2} \mathrm{O}_{4} @ \mathrm{CoFe}_{2}$ core-shell nanoparticles exhibit a nearly smooth hysteresis loop for a 2-nm-thick $\mathrm{CoFe}_{2}$ shell. However, for a 6-nm-thick $\mathrm{CoFe}_{2}$ shell the hysteresis loop resembles the behavior of exchange-spring systems, with clear early shell reversal at positive fields. Furthermore, the core-shell reversal field is nearly constant for a $\mathrm{CoFe}_{2}$ volume fraction ranging from $40 \%$ to $80 \%$.

We have found that the large dipolar field gradients may compensate the exchange energy, allowing large magnetization changes on a small length scale. As in HSCS nanoparticles, this may also be a prominent feature of other nanostructured magnetic systems, such as composites containing hard inclusions in a soft matrix.

We suggest that the present discussion might be helpful for the design of core-shell particles for key applications, such as high-energy-product magnets and magnetic systems for hyperthermia.

\section{ACKNOWLEDGMENTS}

This research was supported by CAPES, FAPERN, and CNPq (A.S.C., Grant. No. 350773, and A.L.D., Grant No. 309676).
[1] A. López-Ortega, M. Estarder, G. Salazar-Alvarez, A. G. Roca, and J. Nogués, Phys. Rep. 553, 1 (2015).

[2] J. Nogués, J. Sort, V. Langlais, V. Skumryev, S. Suriñach, J. S. Muñoz, and M. D. Baró, Phys. Rep. 422, 65 (2005).

[3] J. Nogués and I. K. Schuller, J. Magn. Magn. Mater. 192, 203 (1999).

[4] B. Dieny, V. S. Speriosu, S. S. P. Parkin, B. A. Gurney, D. R. Wilhoit, and D. Mauri, Phys. Rev. B 43, 1297(R) (1991).

[5] B. Dieny, B. A. Gurney, S. E. Lambert, D. Mauri, S. S. P. Parkin, V. S. Speriosu, and D. R. Wilhoit, Patent US5206590 A (1993).

[6] S. S. P. Parkin, K. P. Roche, M. G. Samant, P. M. Rice, R. B. Beyers, R. E. Scheuerlein, E. J. O'Sullivan, S. L. Brown, J. Bucchigano, D. W. Abraham, Yu Lu, M. Rooks, P. L. Trouilloud, R. A. Wanner, and W. J. Gallagher, J. Appl. Phys. 85, 5828 (1999).

[7] S. Parkin, X. Jiang, C. Kaiser, A. Panchula, K. Roche, and M. Samant, Proc. IEEE 91, 661 (2003).
[8] O. Iglesias, A. Labarta, and X. Batlle, J. Nanosci. Nanotechnol. 8, 2761 (2008).

[9] O. Iglesias, X. Batlle, and A. Labarta, J. Phys. Condens. Matter 19, 406232 (2007).

[10] O. Iglesias and A. Labarta, Physica B (Amsterdam, Neth.) 372, 247 (2006).

[11] E. De Biasi, C. A. Ramos, R. D. Zysler, and D. Fiorani, Physica B (Amsterdam, Neth.) 372, 345 (2006).

[12] M. Vasilakaki and K. N. Trohidou, Phys. Rev. B 79, 144402 (2009).

[13] M. H. Wu, Q. C. Li, and J.-M. Liu, J. Phys. Condens. Matter 19, 186202 (2007).

[14] O. Iglesias, X. Batlle, and A. Labarta, J. Phys. D 41, 134010 (2008).

[15] M. Vasilakaki, K. N. Trohidou, and J. Nogues, Sci. Rep. 5, 9609 (2015).

[16] V. Nandwana, G. S. Chaubey, K. Yano, C. Rong, and J. P. Liua, J. Appl. Phys. 105, 014303 (2009). 
[17] J. M. Soares, V. B. Galdino, O. L. A. Conceição, M. A. Morales, J. H. Araújo, and F. L. A. Machado, J. Magn. Magn. Mater. 326, 81 (2013).

[18] J. M. Soares, V. B. Galdino, and F. L. A. Machado, J. Magn. Magn. Mater. 350, 69 (2014).

[19] G. C. P. Leite, E. F. Chagas, R. Pereira, R. J. Prado, A. J. Terezo, M. Alzamora, and E. Baggio-Saitovitch, J. Magn. Magn. Mater. 324, 2711 (2012).

[20] M. Abbas, M. N. B. P. Rao, K. E. A. Aitah, and C. G. Kim, Mater. Lett. 139, 161 (2015).

[21] J. H. Lee, J. T. Jang, J. S. Choi, S. H. Moon, S. H. Noh, J. W. Kim, J. G. Kim, I. S. Kim, K. I. Park, and J. W. Cheon, Nat. Nanotechnol. 6, 418 (2011).

[22] S. Noh, W. Na, J. Jang, J. Lee, E. J. Lee, S. H. Moon, Y. Lim, J. Shin, and J. Cheon, Nano Lett. 12, 3716 (2012).

[23] J. W. Cheon and J. T. Jang, Patent US2011/0151019 A1 (2011).

[24] P. Vargas, D. Altbir, and J. d'Albuquerque e Castro, Phys. Rev. B 73, 092417 (2006).

[25] D. Kechrakos, K. N. Trohidou, and M. Vasilakaki, J. Magn. Magn. Mater. 316, e291 (2007).

[26] G. Margaris, K. N. Trohidou, and J. Nogués, Adv. Mater. 24, 4331 (2012).

[27] See, for instance, http://math.nist.gov/oommf.

[28] A. Grzybowski, E. Gwóźdź, and A. Bródka, Phys. Rev. B 61, 6706 (2000).

[29] M. E. Schabes and H. Neal Bertram, J. Appl. Phys. 64, 1347 (1988).

[30] L. L. Oliveira, J. T. S. Dantas, R. M. Souza, A. S. Carriço, and A. L. Dantas, J. Appl. Phys. 115, 17D130 (2014).

[31] A. L. Dantas, I. Queiroz, Jr., G. O. G. Rebouças, M. G. Dias, and A. S. Carriço, J. Appl. Phys. 113, 17D710 (2013).

[32] T. R. S. Moura, F. F. Oliveira, A. S. Carriço, A. L. Dantas, and G. O. G. Rebouças, J. Appl. Phys. 111, $07 D 116$ (2012).

[33] F. F. Oliveira, T. R. S. Moura, A. S. Carriço, A. L. Dantas, and G. O. G. Rebouças, J. Appl. Phys. 111, 07D102 (2012).

[34] A. S. M. Silva, A. L. Dantas, G. O. G. Rebouças, and A. S. Carriço, J. Appl. Phys. 109, 07D314 (2011).

[35] A. L. Dantas, G. O. G. Rebouças, and A. S. Carriço, IEEE Trans. Magn. 46, 2311 (2010).

[36] P. O. Jubert, IEEE Trans. Magn. 50, 3002004 (2014).

[37] E. C. Stoner and E. P. Wohlfarth, Philos. Trans. R. Soc. London 240, 599 (1948).

[38] N. Poudyal and J. P. Liu, J. Phys. D 46, 043001 (2013).

[39] M. Estrader, A. López-Ortega, S. Estradé, I. V. Golosovsky, G. S. Alvarez, M. Vasilakaki, K. N. Trohidou, M. Varela,
D. C. Stanley, M. Sinko, M. J. Pechan, D. J. Keavney, F. Peiró, S. Suriñach, M. D. Baró, and J. Nogués, Nat. Commun. 4, 2960 (2013).

[40] J. Nogues, D. Lederman, T. J. Moran, and I. K. Schuller, Phys. Rev. Lett. 76, 4624 (1996).

[41] Q. Song and Z. J. Zhang, J. Am. Chem. Soc. 134, 10182 (2012).

[42] H. Zeng, J. Li, Z. L. Wang, J. P. Liu, and S. Sun, Nano Lett. 4, 187 (2004).

[43] F. Liu, J. Zhu, W. Yang, Y. Dong, Y. Hou, C. Zhang, H. Yin, and S. Sun, Angew. Chem., Int. Ed. 53, 2176 (2014).

[44] Y. Zhang, Z. Yang, B. Zhu, S. Chen, X. Yang, R. Xiong, and Yong Liu, J. Alloys Compd. 567, 73 (2013).

[45] M. A. Radmanesh, S. A. S. Ebrahimi, A. Yourdkhani, and H. Khanmohammadi, J. Supercond. Nov. Magn. 25, 2757 (2012).

[46] H. Akbari, S. A. Sebt, H. Arabi, H. Zeynali, and M. Elahi, Chem. Phys. Lett. 524, 78 (2012).

[47] P. Villars, Material Phases Data System (MPDS), Vitznau, Switzerland; SpringerMaterials, http://materials.springer.com/.

[48] M. Y. Rafique, L. Pan, Q. Javet, M. Z. Iqbal, and L. Yang, J. Nanopart. Res. 14, 1189 (2012).

[49] R. R. Kiran, R. A. Mondal, S. Dwevedi, and G. Markandeyulu, J. Alloys Compd. 610, 517 (2014).

[50] M. Y. Rafique, P. L. Qing, Q. Javed, M. Z. Iqbal, Q. H. Mei, M. H. Farooq, G. Z. Gang, and M. Tanveer, Chin. Phys. B 22, 107101 (2013).

[51] M. Mohan, V. Chandra, and S. S. Manoharana, J. Mater. Res. 23, 1849 (2008).

[52] N. Viart, R. S. Hassan, J. L. Loison, G. Versini, F. Huber, P. Panissod, C. Mény, and G. Pourroy, J. Magn. Magn. Mater. 279, 21 (2004).

[53] N. Viart, R. S. Hassan, C. Mény, P. Panissod, C. U. Bouillet, J. L. Loison, G. Versini, F. Huber, and G. Pourroy, Appl. Phys. Lett. 86, 192514 (2005).

[54] A. Juhin, A. L. Ortega, M. Sikora, C. Carvallo, M. Estrader, S. Estradé, F. Peiró, M. D. Baró, P. Sainctavit, P. Glatzel, and J. Nogués, Nanoscale 6, 11911 (2014).

[55] K. L. Krycka, J. A. Borchers, G. S. Alvarez, Alberto L. Ortega, M. Estrader, S. Estradé, E. Winkler, R. D. Zysler, J. Sort, F. Peiró, M. D. Baró, C. C. Kao, and J. Nogués, ACS Nano 7, 921 (2013).

[56] C. M. Souza, A. L. Dantas, I. S. Queiroz, Jr., and A. S. Carriço, J. Appl. Phys. 115, 17D110 (2014).

[57] R. Skomski and J. M. D. Coey, Phys. Rev. B 48, 15812 (1993). 